\title{
Grundlagen der Therapie der chronischen Niereninsuffizienz bei Hund und Katze
}

\author{
Jörg Wolfgang Schäffner
}

\begin{abstract}
Eine frühzeitig begonnene Therapie ist bei der chronischen Niereninsuffizienz wichtig, um die Überlebenszeit des Patienten zu verlängern. Hierbei scheinen die Kontrolle des Serumphosphatspiegels und die Vermeidung oder Reduktion der Proteinausscheidung im Primärharn wichtige Ansatzpunkte zu sein.
\end{abstract}

Die chronische Niereninsuffizienz (CNI) ist eine häufige Erkrankung, vor allem älterer Hunde und Katzen. Die Ursachen für diese chronisch-entzündliche Erkrankung sind bis jetzt nicht vollständig geklärt. Offensichtlich spielt aber eine fortschreitende tubulo-interstitielle Entzündung eine entscheidende Rolle bei der Progression der Erkrankung [1 - 3]. Über die Bedeutung einer rechtzeitigen Diagnostik in Bezug auf eine frühzeitige Therapie und eine längere Überlebenszeit besteht weitgehende Übereinstimmung. Dafür können eine Reihe verschiedener Laborparameter im Serum oder Urin genutzt werden.

Unabhängig von möglichen Grundursachen der chronischen Niereninsuffizienz scheinen 2 Pathomechanismen für das Fortschreiten der Erkrankung und eine schlechte Prognose mitverantwortlich zu sein: eine erhöhte Konzentration von Protein im Urin und ein erhöhter Serumphosphatspiegel. Hier ergeben sich somit 2 wichtige Ansatzpunkte für die Therapie der chronischen Niereninsuffizienz, nämlich die Kontrolle des Serumphosphatspiegels und die Vermeidung/Reduktion der Proteinausscheidung im Primärharn.

\section{Einleitung}

Die chronische Niereninsuffizienz ist eine bei Hunden und Katzen häufig diagnostizierte Erkrankung. Sie wird bei älteren Katzen 2- bis 3-mal häufiger diagnostiziert als bei älteren Hunden. Die Primärursachen dieser progressiven Erkrankung sind bis heute unklar; diskutiert werden neben genetischen Ursachen Infektionserkrankungen, Impfungen, Ernährung, Medikamente oder der Zeitpunkt der Kastration. Alle diese Ursachen erklären kaum die hohe Inzidenz an chronischer Niereninsuffizienz, insbesondere in der Katzenpopulation. Andere offensichtliche Primärursachen wie Erkrankungen der unteren Harnwege durch Inflammation/Infektion (LUTD), Obstruktion durch Steinund Konkrementbildung, erhöhter Blutdruck oder veränderte Gewebeperfusion infolge einer Hyperthyreose oder Herzerkrankung sowie definierte genetische Erkrankun- gen wie polyzystische Nierenerkrankung erklären zwar einzelne konkrete Fälle, sind aber nicht für die Vielzahl von älteren Hunden und vor allem Katzen mit chronischer Niereninsuffizienz verantwortlich.

Sicher scheint zu sein, dass vor allem bei der Katze eine tubulo-interstitielle Nephritis für die fortschreitenden histologischen Veränderungen und den Funktionsverlust des Nierengewebes verantwortlich sind. Eine erhöhte Proteinkonzentration im (Primär-)Harn hat zunächst ja eine glomeruläre Ursache. Durch die Reabsorption der übermäßig ausgeschiedenen Proteine im proximalen Tubulus scheint eine solche Hyperproteinurie ein wichtiger Trigger für das Fortschreiten der tubulären Entzündung und somit der chronischen Niereninsuffizienz zu sein.

Mit großen individuellen Unterschieden kommt es bei Hunden und Katzen im Laufe der chronischen Niereninsuffizienz zu einem Anstieg der Serumphosphat-Konzentration. Der genaue Pathomechanismus wird dabei noch diskutiert. Zahlreiche Autoren machen einen sekundären Hyperparathyreoidismus dafür verantwortlich [4]. Fest steht, dass eine solche Hyperphosphatämie zu einer weiteren Schädigung des funktionellen Nierengewebes und damit zur Progression der chronischen Niereninsuffizienz beiträgt.

Eine Senkung der Hyperproteinurie sowie eine Kontrolle der Serumphosphat-Konzentration scheinen neben anderen Maßnahmen (Proteinrestriktion in der Nahrung, ggf. Rehydratation etc.) Grundpfeiler der Therapie der chronischen Niereninsuffizienz zu sein. 
PRAXISTIPP

Eine frühzeitige Diagnostik der chronischen Niereninsuffizienz ist elementare Voraussetzung für einen frühen und damit erfolgreichen Beginn der Therapie.

Grundlagen der Diagnostik sind andernorts ausführlich beschrieben worden $[1,5]$, daher sollen sie hier nur kurz umrissen werden. Klinisch (Polydipsie, eventuell Anorexie, gerade beim Hund häufig Vomitus, bei der Katze häufig Gewichtsabnahme) wird die chronische Niereninsuffizienz erst zu einem späten Zeitpunkt der Erkrankung offensichtlich. Daher sollte gerade bei älteren Tieren der Fokus auf eine gezielte Laboruntersuchung nierenspezifischer Parameter bei Routinelaboruntersuchungen (Alterscheck) gerichtet werden.

Über die Sensitivität und Spezifität verschiedener Laborparameter wurde viel diskutiert. In Kürze lässt sich feststellen, dass für die Routineuntersuchung folgende Parameter einen Hinweis auf eine chronische Niereninsuffizienz geben:

- wiederholte Bestimmung des Serumkreatinins zu definierten Bedingungen (ein kontinuierlicher Anstieg auch innerhalb der Normwerte ist ein signifikanter Hinweis auf eine entstehende chronische Niereninsuffizienz),

- die Bestimmung der Urindichte (Hypostenurie) oder

- ein erhöhtes symmetrisches Dimethylarginin (SDMA) im Serum.

Darüber hinaus sollte bei Verdacht auf eine chronische Niereninsuffizienz immer der Protein/Kreatinin-Quotient (UPC) im Urin bestimmt werden [1,6].

\section{Grundlagen der Therapie der chronischen Niereninsuffizienz}

Bis jetzt scheint die Pathophysiologie der chronischen Niereninsuffizienz nicht ausreichend verstanden [2,3]. Darüber hinaus haben wir es bei chronischer Niereninsuffizienz von Hund und Katze ohnedies mit einer Erkrankung zu tun, die ätiologisch verschiedene Ursachen haben kann. Angesichts der vielfältigen Ursachen der chronischen Niereninsuffizienz ist eine ätiologische Therapie nur bedingt möglich, beispielsweise bei einer Hyperthyreose, Diabetes mellitus, einer Herzerkrankung oder einer Erkrankung des unteren Urogenitaltrakts. 


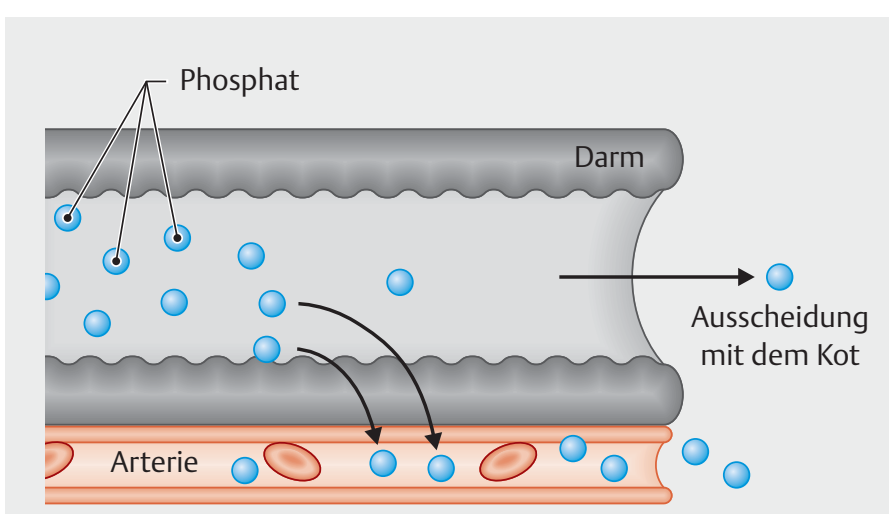

a

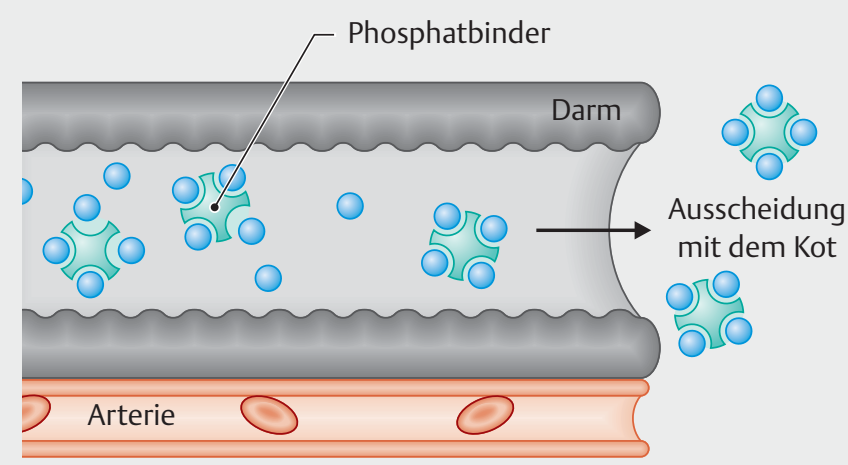

b

- Abb. 1 Mit der Nahrung aufgenommenes Phosphat im Darm. a Phosphatresorption im Darm. b Reduktion der Phosphatresorption im Darm durch Einsatz eines Phosphatbinders und die Ausscheidung mit dem Kot. (c) Thieme Gruppe

\section{PRAXISTIPP}

Sinnvoll und wichtig für eine längere Überlebenszeit und eine bessere Lebensqualität [7] ist die therapeutische Beeinflussung der Faktoren, die für das rasche Fortschreiten der Erkrankung verantwortlich sind.

Neben der unmittelbaren Behandlung von Schüben/akuten Verschlimmerungen einer chronischen Niereninsuffizienz mit Symptomen wie Exsikkose, Vomitus und Kachexie durch Flüssigkeitssubstitution, Antiemetika oder temporärer Zwangsfütterung [1] stehen für das Langzeitmanagement 3 therapeutische Maßnahmen im Vordergrund:

- Eine möglichst phosphatarme, proteinreduzierte (evtl. $\mathrm{Na}^{+}$-reduzierte) Diät.

- Eine Phosphatreduktion durch den Einsatz von Phosphatbindern als Zusatz zur Nahrung.

- Die Verringerung der Hyperproteinurie im Primärharn.

\section{Diät}

Für Hunde und Katzen mit chronischer Niereninsuffizienz stehen eine Reihe kommerzieller Diäten zur Verfügung, die sich zum Teil beträchtlich in Bezug auf Protein-, Phosphat- und $\mathrm{NaCl}$-Gehalt unterscheiden. Gerade bei Katzen bestehen immer wieder erhebliche Unterschiede in der Akzeptanz angebotener Diätfuttermittel.

\section{Merke}

Grundsätzlich gilt, dass eine Katze mit chronischer Niereninsuffizienz besser „normales“ Futter aufnimmt als gar keines.

Denn ein kataboler Zustand mit Abbau körpereigenen Proteins belastet die Niere zusätzlich. Eventuell kann in diesem Fall das gewohnte Futter weiter gefüttert werden, indem Phosphatüberschüsse durch einen gut akzeptierten Phosphatbinder reduziert werden [8].

Ein zu hoher Proteinanteil im Futter kann z. B. durch den Zusatz kohlenhydratreicher Futtermittel wie etwa Kartoffelflocken gesenkt werden. Letzteres funktioniert in der Praxis eher beim Hund.

\section{Reduktion der Hyperphosphatämie}

Unabhängig von den zum Teil kontrovers diskutierten Pathomechanismen besteht Konsens in Bezug auf die Tatsache, dass eine Hyperphosphatämie im Laufe einer chronischen Niereninsuffizienz zu einer weiteren histologischen und funktionellen Schädigung der Niere führt. Diesen Circulus vitiosus gilt es frühzeitig zu erkennen und zu durchbrechen. Allgemein sprechen wir von einer Hyperphosphatämie bei einem Serumphosphat $>6 \mathrm{mg} /$ $\mathrm{dl}$ bei der Katze und >6,5 mg/dl beim Hund [7].

\section{Merke}

Es erscheint jedoch vorteilhaft, bereits ab einer Serumphosphat-Konzentration von $4,5 \mathrm{mg} / \mathrm{dl}$ mit Maßnahmen zur Phosphatreduktion zu beginnen [8].

Für die Katze wurden die anzustrebenden Phosphatkonzentrationen für die 3 festgelegten Stadien der chronischen Niereninsuffizienz in einem Konsensuspaper eindeutig definiert [1]. Fressen Hund oder Katze eine kommerzielle oder selbst zubereitete Nierendiät, ist diese in unterschiedlichem Maße phosphatreduziert. Oft reicht diese Reduktion jedoch bei weiterer Progression der chronischen Niereninsuffizienz nicht mehr aus, um die Serumphosphat-Konzentration im angestrebten Normbereich zu halten, sodass der Einsatz eines Phosphatbinders unerlässlich ist [9]. Grundsätzlich muss festgestellt werden, dass sich der Serumphosphatspiegel ausschließlich durch eine Reduktion der Phosphataufnahme kontrollieren lässt und nicht durch eine erhöhte Ausscheidung der funktionsgestörten Niere. 


\section{physiologische Nierenfunktion}
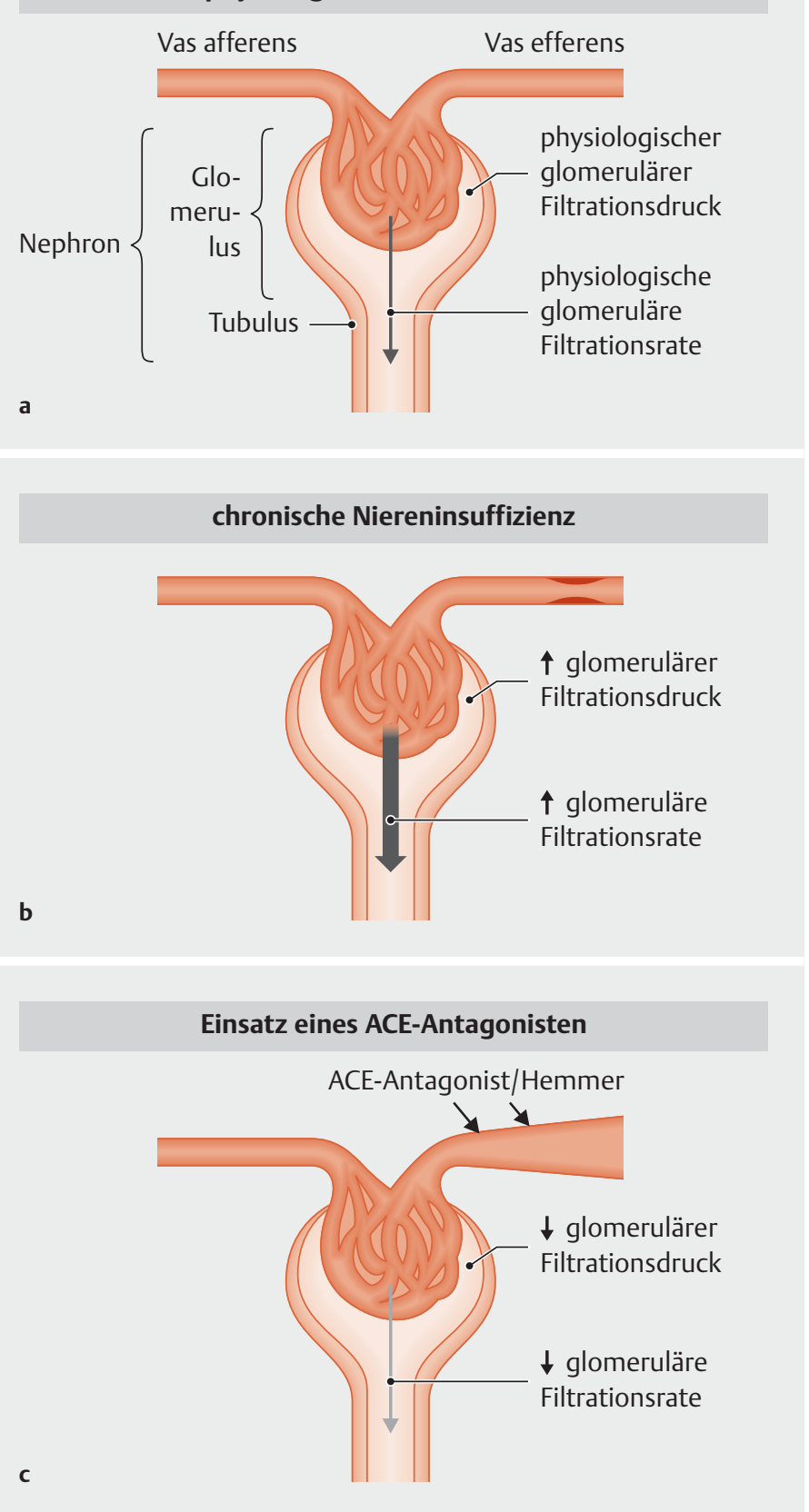

- Abb. 2 Nierenfunktion bei der Katze. a Physiologische Nierenfunktion. b Bei chronischer Niereninsuffizienz: erhöhter glomerulärer Filtrationsdruck und gesteigerte glomeruläre Filtrationsrate; in der Folge kommt es zu einer erhöhten Proteinkonzentration im Primärharn. c Einsatz einen ACE-Antagonisten: Senkung des glomerulären Filtrationsdrucks; in der Folge kommt es zu einem Abfall der glomerulären Filtrationsrate und dadurch zu einer reduzierten Proteinkonzentration im Primärharn. (๑) Thieme Gruppe
Prinzipiell funktioniert eine solche Phosphatreduktion durch ein phosphatarmes Futter oder durch die Bindung des überschüssigen Phosphats im Darm ( $\triangleright$ Abb.1). Phosphatbinder beruhen auf der Reaktion zwischen dem negativ geladenen Phosphat und verschiedenen (meist 2fach positiv geladenen) Kationen, insbesondere Kalzium und Magnesium. Es bildet sich ein unlöslicher Phosphatkomplex im Magen-Darm-Trakt; dieser kann auch in den proximalen Darmabschnitten nicht mehr resorbiert werden.

Den breitesten Einsatz bei Tieren finden Kalziumverbindungen als Phosphatbinder. Im Vergleich mit anderen Phosphatbindern verfügt Kalzium über ein optimales Phosphatbindungsvermögen im leicht sauren Bereich bei $\mathrm{pH} 4$, wie er im Übergang zum Duodenum zu finden ist. Bei einem pH von 2, also im Magen, bindet z. B. Eisen besser als Kalzium. Magnesium dagegen hat sein $\mathrm{pH}-\mathrm{Op}-$ timum für die Phosphatbindung erst im Bereich von 7-8, sodass es erst in den hinteren Darmabschnitten zur vollen Wirkung kommt. Grundsätzlich besteht beim Einsatz von kalziumhaltigen Phosphatbindern das Risiko der Hyperkalzämie [6], bei Magnesiumverbindungen kann es zu vermehrter Ausscheidung dieses Minerals über den Harn kommen. Entscheidend für die Wirksamkeit ist stets die Menge an phosphatbindenden Substanzen, die das Tier freiwillig mit dem Futter aufnimmt. So erweisen sich bestimmte Präparate bei angegebener Dosierung als wenig effizient in der Phosphatbindung [10].

Da auch bei Menschen mit chronischer Niereninsuffizienz die zentrale Rolle der Phosphatreduktion für die Behandlung erkannt wurde, wurden neben Kalzium und Magnesium eine Reihe von weiteren geeigneten Substanzen experimentell und klinisch getestet, die auch bei Hund und Katze Anwendung finden oder fanden. Aluminiumverbindungen binden Phosphat sehr effizient und bewirken eine rasche Reduktion der Serumphosphat-Konzentration. Aluminiumsalze gelten jedoch als neurotoxisch und haben daher ihre Zulassung beim Menschen verloren. Präparate auf Basis der seltenen Erde Lanthan zeigten sich ebenfalls als wirksame Phosphatbinder; ein für Tiere angebotenes Präparat mit Lanthancarbonat wurde jedoch vom Markt genommen. Toxizitätsstudien zeigten hohe Lanthankonzentrationen in zahlreichen Geweben bei Hunden.

Einen neuen Ansatz stellt der Einsatz von Eisenverbindungen zur Phosphatreduktion dar. Bei der Katze hat sich das inzwischen in einem Ergänzungsfuttermittel angebotene Fe-III-Oxid $\left(\mathrm{Fe}_{2} \mathrm{O}_{3}\right)$ als sehr wirksam in der Reduktion der intestinalen Phosphatresorption erwiesen [11]. Fe-III-Oxide werden in extrem geringem Umfang aus dem Darm aufgenommen, sodass auch hohe Eisengaben keine erhöhten Serumeisenspiegel induzieren. Allerdings kann es als Nebenwirkung des Einsatzes zur Diarrhoe kommen, die jedoch bei einschleichender Dosissteige- 
rung ausbleibt. Eine andere beobachtete und durchaus „erwünschte“ Nebenwirkung ist wahrscheinlich der Anstieg des Hämatokrits, obwohl Eisen-III-Oxid kaum resorbiert wird; bedenkt man, dass mittlerweile bei CNIabhängiger Anämie neben der Applikation eines Erythropoetin-Analogons zur Eisensubstitution geraten wird, also eher ein Effekt, den man gern in Kauf nimmt.

\section{Senkung der Hyperproteinurie}

Wie oben dargelegt, werden durch eine vermehrte tubuläre Rückresorption von glomerulär filtriertem Protein Tubulusepithelien geschädigt. Ein hoher Proteingehalt im Primärharn triggert also die Progression der chronischen Niereninsuffizienz $[3,4]$.

\section{Merke}

Bei nachgewiesenem erhöhten UPC sollte der glomeruläre Blutdruck gesenkt werden [5]. 
Dadurch erreicht man eine Reduktion der glomerulären Filtration von Protein in den Primärharn ( $\bullet$ Abb. 2). Leider kommt es aber auch temporär zu einer Senkung der glomerulären Filtrationsrate, was zunächst einen milden Anstieg des Serumkreatinins nach Therapiebeginn nach sich zieht. Langfristig wird durch diese therapeutische Maßnahme die Überlebenszeit jedoch verlängert und die Lebensqualität verbessert.

Für die Katze ist für diese Indikation ein AngiotensinConverting-Enzyme-Antagonist (ACE-Antagonist) zugelassen, für den Hund kann auf zahlreiche zugelassene ACE-Hemmer (ACEI) zurückgegriffen werden. Ob die Kombination von ACEI und ACE-Antagonisten oder sogar die Kombination mit Aldosteron-Antagonisten weitere Vorteile bietet, ist unklar [3].

\section{Weitere mögliche therapeutische Optionen}

Über die erwähnten Standardtherapien hinaus haben sich andere Behandlungsmaßnahmen als sinnvoll erwiesen:

- Therapie der chronischen Anämie: Unabhängig von den kausalen Zusammenhängen bei der Entstehung der Anämie hat sich eine Therapie mit dem Erythropoetin-Analogon Darbepoetin $(1 \mu \mathrm{g} / \mathrm{kg}$ s. c. 1 -mal pro Woche) als sinnvoll erwiesen [12]. Auf die empfohlene zusätzliche Eisensupplementierung kann vermutlich bei der Verwendung eines Eisen-III-Oxid-haltigen Phosphatbinders verzichtet werden.

- Substitution von aktivem Vitamin D3: Nach erfolgreicher Kontrolle der Serumphosphat-Konzentration kann die Substitution von 1,25-Dihydroxycholecalciferol einen zusätzlichen therapeutischen Effekt bieten [10]. Der Parathormonspiegel kann durch die Vitamin-D3-Substitution sinken oder zumindest kann ein Anstieg vermieden werden [13].

- Homöopathische Behandlung: Viele Tierbesitzer verlangen eine zusätzliche homöopathische Therapie der chronischen Niereninsuffizienz oder deren Folgen. Seit längerer Zeit wird hierzu entweder eine fixe Kombination u.a. aus Renes bovis, Viscum album, Equisetum und Veratrum album oder eine Kombinationstherapie aus Coenzymen, Solidago, Ubichinon und Ren suis empfohlen und von vielen Kollegen regelmäßig angewandt.

\section{Kontrolluntersuchungen}

Mindestens monatlich sollten bei klinisch stabilen Patienten Serumkreatininspiegel und Serumphosphat-Konzentration bestimmt werden. Darüber hinaus ist die Kontrolle des (roten) Blutbilds, des UPC sowie des Körpergewichts sinnvoll. Bei Verschlechterung einzelner Parameter sind häufigere Kontrollen zu empfehlen [1]. 


\section{Korrespondenzadresse}

Dr. med. vet. Jörg Wolfgang Schäffner

Fachtierarzt für Kleintiere

Fachtierklinik für Kleintiere im Eichenbach

Lippstraße 18, 77716 Haslach im Kinzigtal

schaeffner.joerg@gmx.de

Literatur

[1] Sparkes AH, Caney S, Chalhoub S et al. ISFM consensus guidelines on the diagnosis and management of feline chronic kidney disease. J Feline Med Surg 2016; 18: 219-239

[2] Chakrabarti S, Syme HM, Brown CA et al. Histomorphometry of feline chronic kidney disease and correlation with markers of renal dysfunction. Vet Pathol 2013; 50: 147-155

[3] Jepson RE. Current understanding of the pathogenesis of progressive chronic kidney disease in cats. Vet Clin North Am Small Anim Pract 2016; 46: 1015-1048

[4] Reynolds B, Lefebvre H. Feline CKD. Pathophysiology and risk factors - what do we know? J Feline Med Surg 2013; 15: 3-14

[5] Steinbach S. Die chronische Nierenerkrankung der Katze. Ein Update über Diagnose, Therapie und Prognose. Komp kleintier 2014; 17-24

[6] Francey T, Müller E. Chronische Nierenerkrankung - Früherkennung und Bedeutung der Phosphate. Vet Spiegel 2010; 3: $107-114$

[7] Roudebush P, Polzin DJ, Adams LG et al. An evidence-based review of therapies for canine chronic kidney disease. I Small Anim Pract 2010; 51: 244-225
[8] Brown SA, Rickertsen M, Sheldon S. Effects of an intestinal phosphorus binder on serum phosphorus and parathyroid hormone concentration in cats with reduced renal function. Intern J Appl Res Vet Med 2008; 6 (3): 155-160

[9] Zentek J, Paßlack N, Kröger S. Pathophysiologische Besonderheiten bei Hunden und Katzen mit chronischer Niereninsuffizienz und Konsequenzen für die Diätetik. Kleintierprax 2009; 54 (7): 393-406

[10] Chew DJ. Chronic kidney disease (CKD) in dogs \& cats - staging and management strategies. Program 1027-30047, AAVSB RACE approved; 2016

[11] Paßlack N, Neumann K, Zentek J. Effekte eines neuen Phosphatbinders auf Eisenoxidbasis auf die renale Phosphatausscheidung bei adulten, gesunden Katzen - eine Pilotstudie Kleintierprax 2014; 59: 58-68

[12] Chalhoub S, Langston CE, Farrelly ]. The use of darbepoetin to stimulate erythropoiesis in anemia of chronic kidney disease in cats: 25 cases. J Vet Intern Med 2012; 26 (2): 363-369

[13] Cortadellas O, Fernández del Palacio MJ, Talavera J et al. Calcium and phosphorus homeostasis in dogs with spontaneous chronic kidney disease at different stages of severity. J Vet Intern Med 2010; 24: 73-79

Bibliografie

DOI https://doi.org/10.1055/s-0044-100323

kleintier konkret 2018; 21: 12-19

c) Georg Thieme Verlag KG Stuttgart · New York

ISSN 1434-9132 